\title{
Anesthetic techniques and recurrence of breast cancer: unanswered questions
}

\author{
Ryungsa Kim ${ }^{1}$, Takanori Kin ${ }^{2}$ \\ ${ }^{1}$ Breast Surgery, Hiroshima Mark Clinic, Hiroshima, Japan; ${ }^{2}$ Departmet of Breast Surgery, Hiroshima City Hospital, Hiroshima, Japan \\ Correspondence to: Ryungsa Kim, MD, PhD. Breast Surgery, Hiroshima Mark Clinic, Hiroshima, Japan. Email: ryu@hbc-center.com. \\ Provenance and Peer Review: This article was commissioned by the editorial office, Gland Surgery. The article did not undergo external peer review. \\ Comment on: Yan T, Zhang GH, Cheng YZ, et al. Effects of anesthetic technique and surgery on myeloid-derived suppressor cells and prognosis in \\ women who underwent breast cancer surgery: a prospective study. Cancer Manag Res 2019;11:5513-22.
}

Submitted Nov 12, 2019. Accepted for publication Nov 24, 2019.

doi: $10.21037 /$ gs.2019.12.20

View this article at: http://dx.doi.org/10.21037/gs.2019.12.20

Over the last decade, a potential link between anesthetic techniques and the recurrence of breast cancer has been an important and controversial issue for anesthesiologists and breast surgeons. This controversy was instigated by a radical retrospective study, which reported that the anesthetic technique employed influenced the recurrence (and hence survival) of cancer in patients that underwent mastectomy with axillary lymph node dissection (ALND) (1). This study by Exadaktylos et al. in Anesthesiology in 2006 compared paravertebral anesthesia (PVA)/analgesia combined with general anesthesia (GA) against GA/ postoperative morphine analgesia. The results suggested that the former resulted in $25 \%$ lower recurrence of breast cancer or metastasis compared to the latter. Surgical stress and anesthesia activate the neuroendocrine paracrine responses of the hypothalamic-pituitaryadrenal (HPA) axis and sympathetic nervous system (SNS), leading to the suppression of cell-mediated immunity (CMI) by releasing neuroendocrine mediators such as catecholamines, cortisol, and cytokines (2). In turn, these mediators promote the progression and metastasis of tumors. It was proposed that the benefit of PVA is related to the afferent transmission pathway of endocrine mediators being blocked. Such mediators, including vascular endothelial growth factor (VEGF), matrix metalloproteinases, and interleukin (IL) 6 and 8, are endogenous regulators that promote tumor growth and angiogenesis, thereby reactivating micrometastasis (3). Therefore, PVA combined with analgesia reduces the recurrence of breast cancer because PVA prevents afferent neurotransmission from the central nervous system via the HPA axis and blocks efferent activation of the SNS through neuroendocrine stress responses during surgery. In contrast, opioids inhibit immune responses, and might support the survival and angiogenesis of tumor cells, as observed in animal and in vitro models (4). Tumor cell growth or death induced by opioids might depend on the drug concentration and/or the duration of treatment. The recurrence of certain cancers in patients using GA in combination with PVA or opioid analgesics might differ due to immunosuppression and the direct effects of opioids on tumor growth and angiogenesis. The benefits of PVA might be directly derived from the opioid savings or by modulating the neuroendocrine responses with PVA. Thus, minimizing the need for volatile anesthetics and opioids or not use them can reduce the recurrence of cancer.

Two important factors affect immunosuppression based on the fact that the anesthetic technique used impacts the recurrence of breast cancer and survival of patients after surgery; specifically surgical stress and the type of general anesthetic. Surgical stress is determined by the extent of the surgical procedure; for instance, whether it is breast-conserving surgery (BCS) or a mastectomy, and whether it involves sentinel lymph node biopsy (SNB) or ALND. GA techniques include volatile and total intravenous anesthesia (TIVA) with propofol, with or without PVA, and the use of morphine and synthetic opioids during surgery. Immunosuppression induced by surgical stress and anesthesia depends on the neuroendocrine responses of the HPA axis and 


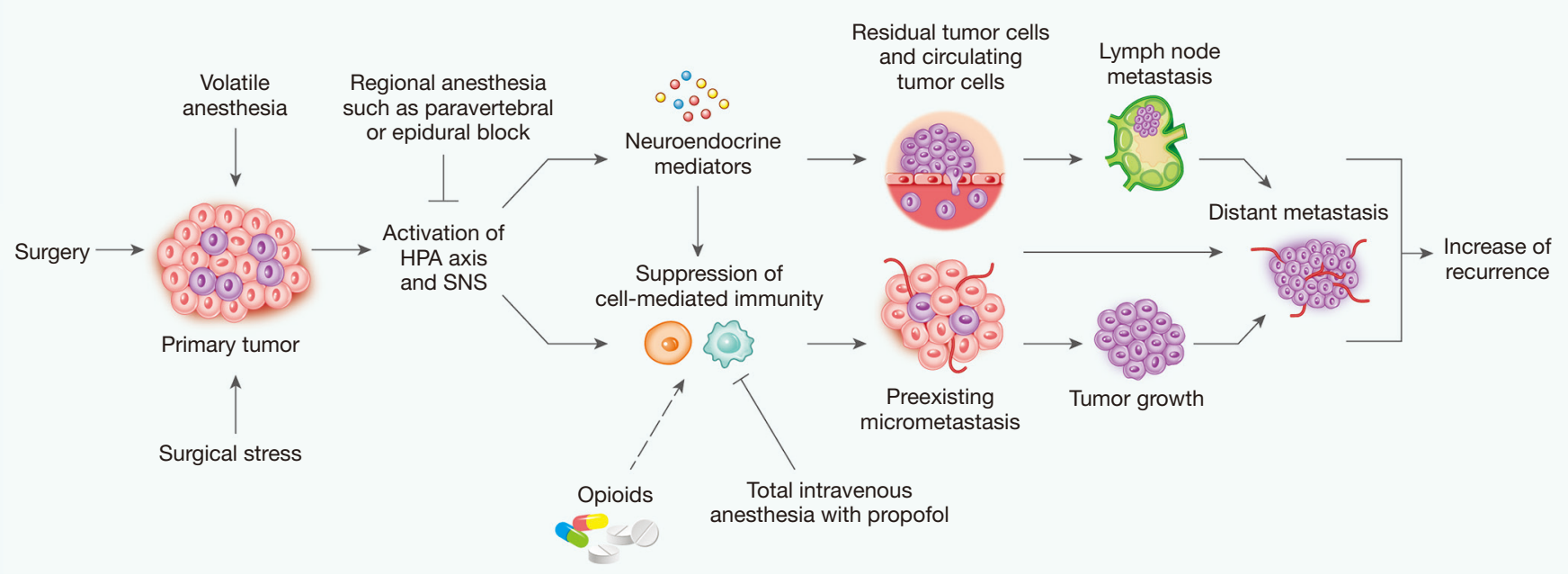

Figure 1 Hypothesis for breast cancer metastasis and recurrence due to immunosuppression by surgery and volatile anesthesia during the perioperative period. Breast cancer surgery and anesthetic agents activate neuroendocrine mediators via the HPA axis and SNS during the perioperative period. These mediators induce several immunosuppressive soluble factors and inflammatory cytokines, which promote the progression and metastasis of tumors, exacerbating the recurrence of breast cancer. Combined regional anesthesia, such as paravertebral or epidural block with propofol, decreases volatile anesthesia and opioid-induced immunosuppression. HPA, hypothalamic-pituitary-adrenal; SNS, sympathetic nervous system. This figure is a modification from the original figure published in Kim R. J Transl Med 2018;16:8.

SNS, because neuroendocrine mediators regulate the biology of tumor progression and might promote overt tumor metastasis due to preexisting micrometastasis or circulating tumor cells (Figure 1). In fact, volatile anesthetics, including morphine and synthetic opioids (but not propofol), have been reported to suppress natural killer (NK)- and T cell-mediated CMI in numerous clinical and animal studies (5-7). Volatile anesthetics impair the functioning of many immune cells such as NK cells and T cells. Volatile anesthetics also stimulate hypoxia-inducible factor $1 \alpha$ and phosphoinositide 3-kinase-Akt signaling pathway, in addition to having antiapoptotic properties. These properties potentially promote the growth of tumor cells in situations where residual disease is minimal (8). In contrast, the use of propofol for TIVA might have protective effects through its anti-inflammatory, antioxidant properties, and its preservation of NK and T cell function $(9,10)$.

Despite the documented role of surgical stress and anesthetic-induced immunosuppression on the recurrence of breast cancer, the benefits of a particular anesthetic technique have not been validated in prospective clinical studies assessing recurrence of breast cancer and survival of patients after surgery. Some retrospective studies have shown that regional anesthesia (RA) combined with PVA or propofol decreases the recurrence of breast cancer and increases the survival of patients when compared with the use of volatile anesthetics (1,11-12). However, other studies do not support this conclusion (13-16). These earlier retrospective studies suggest limited benefits of a particular anesthetic technique in reducing the recurrence of breast cancer. Importantly, a recent prospective study by Yan et al. reported that sevoflurane- and propofol-based anesthesia are not significantly different in terms of their effects on myeloid-derived suppressor cells (MDSCs) and prognosis after breast cancer surgery, including BCS and mastectomy (17). Mastectomy with high surgical stress has lower MDSC levels compared to BCS, but the prognosis was not significantly different, despite over $60 \%$ of patients who had mastectomy in both groups. This is consistent with the same prognosis for patients receiving mastectomy and BCS with radiotherapy. Postoperative levels of MDSCs were not significantly different between sevoflurane and TIVA with propofol; however, the ratios of MDSC subtypes were correlated with tumor stage. MDSCs are immunosuppressive components that promote the growth of tumors and inhibit $\mathrm{CMI}$ in the tumor microenvironment. MDSCs are heterogeneous populations of immature neutrophils, monocytes, and dendritic cells that derive from 
bone marrow. They are stimulated by tumor-derived soluble factors (such as VEGF), and accumulate around the tumor to form an immunosuppressive network that promotes the growth and metastasis of tumors (18). Although this study did not show a survival benefit of propofol versus sevoflurane for peripheral MDSCs, there are limitations to drawing conclusions. One limitation is that MDSCs might be stimulated in parallel with tumor growth and metastatic processes, rather than being enhanced by certain anesthetics (such as sevoflurane and propofol). Other limitations are the small number of samples, a short follow-up period of about 3 years after surgery, and a lack of CMI analysis. On the other hand, it is conceivable that this study excludes both the effect of opioids and the use of propofol for induction of inhalation anesthesia on immune response. However, the recurrence of breast cancer appears to be less likely in patients that receive TIVA compared to sevoflurane. Nonetheless, a long-term follow-up of at least 10 years is required to reach definitive conclusions.

To confirm the benefits of RA with propofol in reducing the recurrence of breast cancer, a large, prospective, and randomized controlled trial was initiated in 2007, as an international multicenter study of breast cancer surgery comparing paravertebral and epidural block with propofol or GA against GA with sevoflurane and opioids (NCT00418457). A feature of the study design was that breast cancer surgery includes both BCS and mastectomy; however, ALND was also a criterion for enrollment. The results of the prospective randomized study have been recently published, showing that no difference in recurrence was observed for RA-analgesia with PVA and propofol versus GA with sevoflurane and opioid analgesia (19). However, it is premature to conclude that a particular anesthetic technique could reduce the recurrence of breast cancer. This is because the median follow-up period is still short (median: 3-years; maximum: around 7 years), even though the number at risk in the RA-anesthesia group is lower than that in the GA group. The potential for reducing the recurrence of breast cancer by using RA-anesthesia with propofol remains in the subgroup, especially in Asian and the BCS group.

In relation to the selection of anesthetic technique, except for some patients with large breasts, most BCS with axillary management (including SNB and ALND) can be performed by day surgery at an outpatient clinic using local anesthesia with lidocaine and intravenous anesthesia with propofol and/or sedation with midazolam without opioids, and without tracheal intubation $(20,21)$. Thus, GA with tracheal intubation is not necessary for BCS. Rather, using lidocaine, while maintaining spontaneous breathing with low-dose intravenous anesthesia with propofol and deep sedation, could help reduce the recurrence of breast cancer. This approach might prevent patients entering a state of immunosuppression, maintaining them in a state close to normal physiological conditions. BCS under local anesthesia and deep sedation and/or intravenous anesthesia with propofol results in a recurrence rate of around 5\% (20,21). In comparison, the recurrence of breast cancer typically tends to be about $15-20 \%$, based on a follow-up period of 10 years. Thus, maintaining spontaneous breathing using local anesthesia and intravenous anesthesia and/ or deep sedation without GA is less immunosuppressive, resulting in a lower recurrence of breast cancer. In fact, changes to immune parameters (including the CD4/8 ratio, peripheral NK cell activity, and IL-6) in patients undergoing local anesthesia and intravenous anesthesia/ deep sedation is likely lower when compared to patients receiving GA with sevoflurane or TIVA with propofol for BCS (22). Thus, maintaining normal physiological conditions, as much as possible, during anesthesia for BCS might carry a survival advantage for breast cancer patients.

Finally, at present, it is not possible to make definitive statements on the benefits of certain types of anesthesia to prevent the recurrence of breast cancer after surgery. It is possible that the choice of anesthetic technique during the perioperative period does affect the long-term outcome. A particular anesthetic technique that maintains spontaneous breathing without using general endotracheal anesthesia and opioids in BCS might provide survival benefits by reducing the recurrence of breast cancer. Further prospective randomized studies comparing nontracheal and endotracheal anesthesia in BCS are vital in the future to facilitate scientifically informed decisions are made for operative procedures.

\section{Acknowledgments}

The Figure 1 is a modification from the original figure published by Springer Nature in "Kim R. Effects of surgery and anesthetic choice on immunosuppression and cancer recurrence. J Transl Med 2018;16:8". The authors would like to thank Springer Nature for the reproduction of the figure.

Funding: None 


\section{Footnote}

Conflicts of Interest: Both authors have completed the ICMJE uniform disclosure form (available at http://dx.doi. org/10.21037/gs.2019.12.20). The authors have no conflicts of interest to declare.

Ethical Statement: The authors are accountable for all aspects of the work in ensuring that questions related to the accuracy or integrity of any part of the work are appropriately investigated and resolved.

Open Access Statement: This is an Open Access article distributed in accordance with the Creative Commons Attribution-NonCommercial-NoDerivs 4.0 International License (CC BY-NC-ND 4.0), which permits the noncommercial replication and distribution of the article with the strict proviso that no changes or edits are made and the original work is properly cited (including links to both the formal publication through the relevant DOI and the license). See: https://creativecommons.org/licenses/by-nc-nd/4.0/.

\section{References}

1. Exadaktylos AK, Buggy D J, Moriarty DC, et al. Can anesthetic technique for primary breast cancer surgery affect recurrence or metastasis? Anesthesiology 2006;105:660-64.

2. Horowitz M, Neeman E, Sharon E, et al. Exploiting the critical perioperative period to improve long-term cancer outcomes. Nat Rev Clin Oncol 2015;12:213-26.

3. Kim R. Effects of surgery and anesthetic choice on immunosuppression and cancer recurrence. J Transl Med 2018;16:8.

4. Sacerdote P, Limiroli E, Gaspani L. Experimental evidence for immunomodulatory effects of opioids. Adv Exp Med Biol 2003;521:106-16.

5. Markovic SN, Knight PR, Murasko DM. Inhibition of interferon stimulation of natural killer cell activity in mice anesthetized with halothane or isoflurane. Anesthesiology 1993;78:700-6.

6. Tavare AN, Perry NJ, Benzonana LL, et al. Cancer recurrence after surgery: direct and indirect effects of anesthetic agents. Int J Cancer 2012;130:1237-50.

7. Deegan CA, Murray D, Doran P, et al. Anesthetic technique and the cytokine and matrix metalloproteinase response to primary breast cancer surgery. Reg Anesth Pain Med 2010;35:490-5.
8. Stollings LM, Jia LJ, Tang P, et al. Immune modulation by volatile anesthetics. Anesthesiology 2016;125:399-411.

9. Inada T, Kubo K, Shingu K. Promotion of interferongamma production by natural killer cells via suppression of murine peritoneal macrophage prostaglandin $\mathrm{E}_{2}$ production using intravenous anesthetic propofol. Int Immunopharmacol 2010;10:1200-8.

10. Desmond F, McCormack J, Mulligan N, et al. Effect of anaesthetic technique on immune cell infiltration in breast cancer: A follow-up pilot analysis of a prospective, randomised, investigator-masked study. Anticancer Res 2015;35:1311-9.

11. Koonce SL, Mclaughlin SA, Eck DL, et al. Breast cancer recurrence in patients receiving epidural and paravertebral anesthesia: a retrospective, case-control study. Middle East J Anaesthesiol 2014;22:567-71.

12. Lee JH, Kang SH, Kim Y, et al. Effects of propofol-based total intravenous anesthesia on recurrence and overall survival in patients after modified radical mastectomy: a retrospective study. Korean J Anesthesiol 2016;69:126-32.

13. Starnes-Ott K, Goravanchi F, Meininger JC. Anesthetic choices and breast cancer recurrence: a retrospective pilot study of patient, disease, and treatment factors. Crit Care Nurs Q 2015;38:200-10.

14. Tsigonis AM, Al-Hamadani M, Linebarger JH, et al. Are cure rates for breast cancer improved by local and regional anesthesia? Reg Anesth Pain Med 2016;41:339-47.

15. Kairaluoma P, Mattson J, Heikkilä P, et al. Perioperative paravertebral regional anaesthesia and breast cancer recurrence. Anticancer Res 2016;36:415-8.

16. Yoo S, Lee HB, Han W, et al. Total intravenous anesthesia versus inhalation anesthesia for breast cancer surgery: A retrospective cohort study. Anesthesiology 2019;130:31-40.

17. Yan T, Zhang GH, Cheng YZ, et al. Effects of anesthetic technique and surgery on myeloid-derived suppressor cells and prognosis in women who underwent breast cancer surgery: a prospective study. Cancer Manag Res 2019;11:5513-22.

18. Kim R, Emi M, Tanabe K, et al. Tumor-driven evolution of immunosuppressive networks during malignant progression. Cancer Res 2006;66:5527-36.

19. Sessler DI, Pei L, Huang Y, et al. Recurrence of breast cancer after regional or general anaesthesia: a randomised controlled trial. Lancet 2019;394:1807-15.

20. Kim R, Kawai A, Wakisaka M, et al. Outcomes of outpatient breast cancer surgery at a private breast clinic. Breast J 2018;24:628-32. 
21. Kim R, Kawai A, Wakisaka M, et al. Outpatient surgery for breast cancer: Experience and outcome in 425 consecutive patients in a private breast clinic. Ann Surg Oncol 2019;26:262.

Cite this article as: $\operatorname{Kim} \mathrm{R}$, Kin T. Anesthetic techniques and recurrence of breast cancer: unanswered questions. Gland Surg 2020;9(2):490-494. doi: 10.21037/gs.2019.12.20
22. Kim R, Kawai A, Wakisaka M, et al. Differences in immune response to anesthetics used for day surgery versus hospitalization surgery for breast cancer patients. Clin Transl Med 2017;6:34. 